\title{
Correction to: Update on lymphoproliferative disorders of the gastrointestinal tract: disease spectrum from indolent lymphoproliferations to aggressive lymphomas
}

\author{
Santiago Montes-Moreno ${ }^{1} \cdot$ Rebecca L. King ${ }^{2} \cdot$ Ilske Oschlies $^{3} \cdot$ Maurilio Ponzoni $^{4} \cdot$ John R. Goodlad ${ }^{5}$. \\ Snjezana Dotlic ${ }^{6}$. Alexandra Traverse-Glehen ${ }^{7} \cdot$ German Ott $^{8} \cdot$ Judith A. Ferry $^{9} \cdot$ Maria Calaminici $^{10}$ (D)
}

Published online: 21 February 2020

(C) Springer-Verlag GmbH Germany, part of Springer Nature 2020

\section{Correction to: Virchows Archiv \\ https://doi.org/10.1007/s00428-019-02704-8}

This erratum is meant to address the error in which the names of one of the authors of the manuscript, was incorrect. Author assumes full responsibility for this error.

The original article has been corrected.

The online version of the original article can be found at https://doi.org/ $10.1007 / \mathrm{s} 00428-019-02704-8$

Maria Calaminici

marie.calaminici@nhs.net

1 Anatomic Pathology Service and Translational Hematopathology Lab, Hospital Universitario Marques de Valdecilla/IDIVAL, Santander, Spain

2 Division of Hematopathology, Mayo Clinic, Rochester, MN, USA

3 Department of Pathology, Hematopathology Section and Lymph node Registry, University Hospitals Schleswig-Holstein, Christian-Albrecht-University, Kiel, Germany

4 Ateneo Vita-Salute San Raffaele and Pathology Unit, San Raffaele Scientific Institute, Milan, Italy

5 Department of Pathology, Queen Elizabeth University Hospital, Glasgow, UK
6 Department of Pathology and Cytology, University Hospital Centre Zagreb, Zagreb, Croatia

7 Department of Hematopathology, Hospices Civils de Lyon/Université Lyon, Lyon, France

8 Department of Clinical Pathology, Robert-Bosch-Krankenhaus, Stuttgart, Germany

9 Department of Pathology, Massachusetts General Hospital and Harvard Medical School, Boston, MA, USA

10 Department of Cellular Pathology, SIHMDS, Barts Health NHS Trust and Centre for Haemato-Oncology, Barts Cancer Institute, London, UK 